\title{
Proyecto mecatrónico de brazo robot cartesiano integrado a una celda de almacenamiento y recuperación automatizada AS / RS de un Sistema Flexible de Manufactura FMS
}

\author{
Jaime Humberto Carvajal Rojas \\ Ph. D. En Ingeniería Mecánica, UNICAMP, SP, Brasil \\ Área de concentración Mecatronica, Robótica y \\ Control de sistemas mecánicos \\ Magister en Sistemas Automáticos de Producción, UTP, Colombia \\ Ingeniero Mecánico, UFPS, Colombia \\ Profesor investigador de Ingeniería Mecatrónica \\ Universidad Santo Tomas de Bucaramanga, Colombia \\ jhcarvajalr@yahoo.com
}

\author{
Rubén Darío Godoy Hernández \\ Ingeniero de Diseño y Automatización Electrónica \\ Universidad de La Salle, Bogotá, Colombia \\ ruben.godoy@gmail.com \\ Willy Rodríguez Quintero \\ Ingeniero de Diseño y Automatización Electrónica \\ Universidad de La Salle, Bogotá, Colombia \\ willyroq@hotmail.com
}

\begin{abstract}
Resumen - Este trabajo es parte de una investigación mayor y presenta modelaje, diseño, simulación e integración de un robot cartesiano para el posicionamiento de piezas en una celda de almacenamiento y recuperación automatizado AS/RS del proyecto sistema flexible de manufactura FMS orientado a manufactura integrado por computador

AS/RS cell are configured by Cartesian robot arm, a vision system in 3D with Artificial Intelligence Al for sensing and codification prismatic parts and cylinder parts, a codification panel for storage and an automatically material handling system AMHS. This AMHS to compose the AS/RS cell into the FMS.
\end{abstract} CIM; en proceso de desarrollo para la enseñanza de tecnologías avanzadas de manufactura AMT. La metodología de modelaje, diseño, simulación e integración del sistema mecatrónico robotizado se desarrolla aplicando conceptos de ingeniería concurrente en ambiente virtual antes de su implementación en ambiente real. La celda de almacenamiento y recuperación automatizada AS/RS está constituida por un robot cartesiano, un sistema de visión artificial en 3D con software inteligente para identificación y clasificación de partes prismáticas y cilíndricas, un panel de almacenamiento y una cinta transportadora que integra físicamente esta celda con las otras celdas del FMS. El robot cartesiano tiene tres grados de libertad, cada articulación es prismática, para el desplazamiento del primer eje se aplica una transmisión flexible por correa dentada conectada a un motor paso a paso. Para el desplazamiento en el segundo eje se aplica una transmisión rígida por tornillo de bolas actuado por un motor paso a paso. Para el desplazamiento en el tercer eje se utiliza un cilindro electro neumático. Y el posicionamiento codificado del elemento terminal, End Of Arm EOA, se realiza con una garra de dos dedos.

Palabras clave-FMS, Mecatrónica, Modelamiento, Diseño, Simulación, Robótica.

\footnotetext{
Abstract- This work develop modelling, design, simulation and integration of a Cartesian robot arm for positioning parts into of an AS/RS cell into the FMS toward CIM for teaching AMT in virtual environment. The methodology is based in virtual environment before implementation in real environment applying Concurrent Engineering CE. The
}

Keywords- FMS, Mechatronic, Modeling, Design, Simulation, Robotics.

\section{INTRODUCCIÓN}

La Federación Internacional de Robótica (IFR International Federation of Robotics) y la Comisión Económica de las Naciones Unidas para Europa (UNECE United Nations Economic Commission for Europe) definen robot industrial con la Norma ISO 8373 como: Un manipulador programable, reprotrolado, con tres o más ejes, uno fijo y otro móvil, para aplicaciones de automatización industrial. [14].

El proyecto interdisciplinario mecánico y electrónico, también denominado proyecto mecatrónico de robots industriales tiene dos opciones: (1) diseño mecatrónico de un nuevo robot para una necesidad específica o (2) selección del robot de una base de datos comercial. La primera opción significa aplicar la metodología de diseño mecatrónico y la segunda opción significa seleccionar el modelo de una base de datos de acuerdo a unos criterios mínimos. En este proyecto, la metodologramable, multipropósito, automáticamente con- 
gía de diseño mecatrónico se basa en ingeniería concurrente (CE Concurrente Engineering) en lugar de secuencial, resultando una aproximación al diseño de productos más sinérgicos y en los sistemas CAD (CAD Computer Aided Design) para el modelamiento de la configuración y simulación del movimiento [3].

El sistema de almacenamiento y recuperación automatizados (AS/RS Automated Storage/Retrieval System) se define como un sistema que realiza operaciones de almacenamiento y recuperación con velocidad y precisión bajo un grado definido de automatización. Cada AS/RS tiene una o más estaciones de entrada/salida donde los materiales son entregados al sistema de almacenamiento o movidos hacia fuera. Las estaciones de entrada/salida (Pickup-and-deposit P\&D station) en terminología AS/RS, pueden ser sistemas automatizados de manejo de materiales como cintas transportadoras o vehículos guiados automáticamente (AGV Automatically Guided Vehicle System). [5].

Ingeniería Concurrente CE. En la última década del siglo XX, la CE emergió como una tendencia en el diseño y manufactura automatizados de nuevos productos en FMS y ha evolucionado a través de la investigación. Recién surgida, los investigadores propusieron diferentes definiciones, algunos la homologan a la ingeniería simultánea (SE Simultaneous Engineering), los diseñadores industriales como Evans [4] afirman que es un método de diseño para la manufactura y ensamblaje; investigadores como Harley y Mortimer [7] la definen como la aplicación del análisis de diseño antes de la producción; Parsaei y Sullivan [11] consideran que su implementación debe apoyarse en el empleo del computador digital en el diseño y la manufactura; Ishii [8] afirma que la CE generalmente se ha reconocido como una práctica de diseño y manufactura, que considera varios valores de ciclos de vida para un producto desde su etapa de diseño y abarca otros aspectos como facilidad de manufactura, facilidad de ensamblaje y funcionabilidad. Shina [12] define la CE como la integración más temprana posible de todos los conocimientos de la fábrica, en recursos, experiencia en el diseño, investigación, mercadeo, manufactura y ventas para la creación de un nuevo producto, con alta calidad, y bajo costo para com- placer las expectativas de los clientes. Y Yazdani [15] propone una definición sintetizada de la CE, como la concurrencia o superposición del diseño y desarrollo de productos y procesos. Con base en los anteriores conceptos, puede considerarse la CE como sinónimo de un buen equipo de trabajo interdisciplinario y multidisciplinario, con buenas relaciones y comunicaciones entre las funciones en cada departamento o especialidad, cuyo objetivo es reducir los tiempos en el diseño y la manufactura por superposición de actividades. Para crear un ambiente en donde la CE sea puesta en práctica es necesario considerar los siguientes atributos. [3]:

(1) Disponer de un equipo de trabajo multidisciplinario.

(2) Que el equipo multidisciplinario trabaje como equipo interdisciplinario.

(3) Que el equipo de trabajo opere en red computadorizada para el intercambio de información.

(4) El equipo de trabajo debe tener el poder de tomar decisiones de acuerdo a su conocimiento y experiencia.

(5) Lo anterior es solamente posible en una organización empresarial horizontal en donde la integración es posible, las comunicaciones son claras, y el personal está directamente involucrado en la ejecución del trabajo y programas.

La filosofía de la CE se centra en el hecho que los grupos de expertos que se integran; manejan y toman decisiones en conjunto respecto al diseño del producto. Groover [5] establece que la CE es posible por la adecuada información, coordinación y cooperación entre los grupos de especialistas que integran la organización de un proyecto. El empleo eficiente de la CE en el proceso de diseño y manufactura, permite el logro de varios objetivos, entre los que se tienen:

(1) Diseño para la manufactura y el ensamblaje.

(2) Diseño para la calidad.

(3) Diseño para ciclo de vida predeterminado.

(4) Diseño para minimizar costos. 
En el diseño mecatrónico hay integración sinérgica de los sistemas, mecánicos, sistemas eléctricoselectrónicos, y sistemas computarizados como un todo, en donde los diseñadores de cada área intervienen en todas las etapas el diseño. Esta sinergia es generada por la combinación correcta de los parámetros, de tal manera, que el diseño final del producto es tan bueno como la suma de las partes que lo componen. Los productos mecatrónicos muestran característica de funcionamiento tan bueno, imposible de lograr sin esta combinación sinérgica.

FIG. 1. Automated storage and retrieval system AS/RS. World robotics [14]

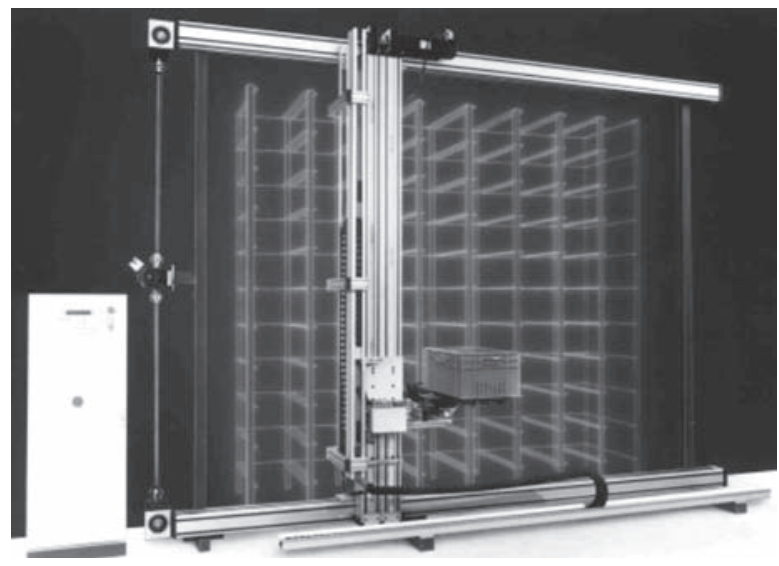

Fuente: Los autores

Sistemas CAD. Los sistemas CAD son usados en el proyecto mecatrónico para el modelo gráfico y simulación del movimiento del robot en 3D, están constituidos por comandos y su flexibilidad les permite encontrar variantes para crear y editar los modelos geométricos. Tienen tres tipos de herramientas para los modelos: (1) modelos en mallas, (2) modelos en superficies y (3) modelos en sólidos. Los modelos con sólidos primitivos son los más empleados y las operaciones booleanas son el método para obtener sólidos compuestos. Con el comando UNION dos o más sólidos se unen para configurar uno solo, absorbiendo la parte común o superpuesta. Se pueden unir sólidos que no se cortan, o sea, sólidos separados y sin contacto. Con el comando SUBSTRACT se obtiene sólidos después de substraer un conjunto de sólidos a otro conjunto de sólidos. Con el comando INTERSECT se obtiene un nuevo sólido configurado con la parte común o intersección de los sólidos interceptados.

\section{METOdOLOGÍA}

\section{A. Diseño mecatrónico de robots}

La metodología de diseño mecatrónico de robots industriales integra tres tareas fundamentales: Modelamiento, Simulación e Integración. Estas tareas interactúan durante todo el proceso de diseño y de manufactura, se ayudan mutuamente y se intercambian datos para hacer ajustes y optimizaciones. Esta metodología está basada en la aplicación de la ingeniería concurrente CE, los sistemas CAD y la ingeniería asistida por computador CAE (CAE Computer Aided Engineering)

FIG. 2. Metodología de diseño mecatrónico de robots industriales.

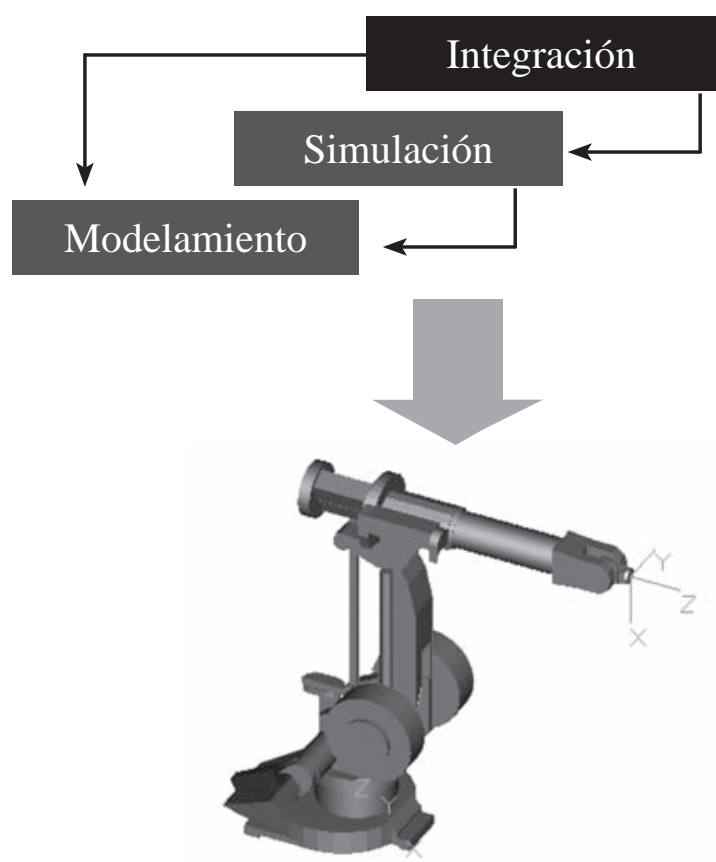

Fuente: Los autores

La tarea de modelamiento toma como base el modelo matemático de la cinemática y de la dinámica del robot, es decir, el conjunto de ecuaciones diferenciales ordinarias que representan el comportamiento del movimiento del robot. Estas ecuaciones diferenciales de orden $\mathrm{n}$ son no-lineales, por lo que hay que darles un tratamiento con base en las series de expansión de Taylor para linealizarlas, a continuación aplicar la transformada de Laplace y finalmente manipular la función de transferencia en forma de diagrama de bloques. En algunos casos se emplea la ecuación de es- 
tado. El modelamiento significa también la representación gráfica en sistema CAD de la configuración del robot. La tarea de simulación analiza el comportamiento de la cinemática del robot y de las diferentes estrategias de control para seleccionar la de mejor comportamiento. La tarea de integración significa el acoplamiento de sistemas mecánicos, eléctricos-electrónicos y computarizados para configurar un nuevo producto mecatrónico. El diagrama de la Figura 3, muestra en bloques la metodología de diseño mecatrónico de robots industriales para el desarrollo de estas tres tareas.

\section{B Modelamiento}

Antes de modelamiento en sistema CAD y el modelamiento de la cinemática del robot, pueden considerarse dos tareas preliminares descritas en los literales i e ii: reconocimiento de una necesidad, diseño conceptual y especificaciones funcionales.

(i) Reconocimiento de una necesidad. Los robots industriales tienen aplicación en los FMS y en los FAS, en donde se dese mpeñan fundamentalmente para el posicionamiento de piezas a ser mecanizadas en máquinas CNC y para el posicionamiento sobre los sistemas de transporte. También, se emplean en el posicionamiento de piezas a ser ensambladas. Estas son las áreas de mayor aplicación de robots en ambientes de manufactura. En menor escala los robots se emplean para efectuar funciones de manufactura como soldadura, pintura, etc. Entonces, inicialmente hay que definir el ambiente de manufactura o de ensamblaje del robot, considerar su estructura adecuada y calcular su volumen o espacio de trabajo.

(ii) Diseño conceptual y especificaciones funcionales. Definido el ambiente de manufactura o de ensamblaje del robot y su tarea, se especifican sus condiciones de funcionamiento, distancias entre puntos de posicionamiento, velocidad y aceleración en los movimientos, cargas a transferir, aproximación a la estructura definitiva, análisis de materiales para los elementos, análisis de la sección de los elementos, análisis de esfuerzos, ubicación de los motores y transmisiones, diseños de guías para articulaciones prismáticas, establecer rango de las variables en las articulaciones, planear tamaño del robot, y cálculo del espacio de trabajo. Con todos estos factores se llega a un bosquejo esquemático del robot requerido.

(iii) Modelo matemático. El modelo matemático, también llamado modelo de la dinámica del robot, es un conjunto de ecuaciones diferenciales ordinarias con las cuales se puede predecir el comportamiento del robot. Implica, asignación de las coordenadas de las articulaciones, obtención de los parámetros de Denavit-Hartemberg, análisis de la cinemática directa, análisis de la cinemática inversa, cálculo del Jacobiano geométrico y análisis de la dinámica por con la formulación de Lagrange Euler o con la formulación de Newton - Euler.

(iv) Modelo y diseño estructural con sistema CAD y CAE. Diseño estructural significa definir la estructura básica del brazo robot, seleccionar los materiales de sus elementos, definir la sección de los elementos, definir el mecanismo en las articulaciones, diseñar la muñeca en donde se acopla la mano o herramienta del robot, analizar las cargas estáticas/dinámicas, analizar los momentos y las inercias. También, diseño estructural implica configurar un modelo virtual mediante el sistema CAD y análisis con sistema CAE.

(v) Selección de motores, transmisiones, sensores y manos. Conocido el modelo de la dinámica del robot, se pueden calcular torques y fuerzas en las articulaciones. Con esta información se seleccionan los motores de catálogos comerciales y se diseña o seleccionan las transmisiones. Al llegar a este paso ya se tienen definidas las variables a controlar, por lo que se pueden seleccionan los sensores correspondientes. Conocidos los sensores puede obtenerse su modelo matemático para representarlo en diagrama de bloques y puedan integrarse al sistema de control. En el paso (i) se ha definido la función del robot y ahora se procede a seleccionar o diseñar el mecanismo de la mano o a seleccionar la herramienta de trabajo.

(vi) Ampliación del modelo matemático. Con los modelos matemáticos de los componentes calculados hasta ahora se puede organizar un solo modelo matemático de todo el sistema del robot; incluido el modelo matemático de los actuadores, transmisiones y sensores. También, en este 
modelo matemático pueden incluirse el efecto de las fricciones en las articulaciones y la influencia de perturbaciones en el sistema para obtener un modelo matemático total, que sirve de base para estudiar la estrategia de control.

(vii) Diseño del sistema de control. El modelo matemático resultante se manipula con operadores diferenciales, transformadas de Laplace y Series de Taylor. La función de transferencia obtenida, que representa el modelo, se simula mediante acciones básicas de control. A partir de este comportamiento inicial se establece la estrategia de control: control de torque, control de fuerza, control adaptativo, control robusto, control robustoadaptivo y otros sistemas de control avanzado. Se busca la estrategia de control que satisfaga los requerimientos funcionales y resulte más económica.

(viii) Optimización del diseño. Se realizan las pruebas necesarias y se ajustan los parámetros y variables del robot para maximizarlos o minimizarlos.

\section{Simulación}

El modelo del prototipo en sistema CAD y el modelo matemático de la cinemática son base para observar el comportamiento del robot antes de implantarse en la realidad y hacer los ajustes que correspondan.

(ix) Simulación gráfica. La simulación gráfica significa la visualización en una estación computarizada de la animación del sistema de control del robot industrial para conocer su comportamiento ante diferentes acciones de control. Para el efecto se emplea software especializado de simulación de robots.

(x) Simulación real. La simulación en tiempo real significa el empleo de dispositivos en lazo cerrado (Hardware-in-loop-simulation) como un subsistema que ejecuta la acción en tiempo real por medio de una tarjeta que reemplaza parte del modelo físico del robot en interface con los motores y sensores. El modelo resultante es parte matemá- tico y parte real; es necesario que se encuentren sincronizadas. Este subsistema también se conoce como simulación con interface-en-tiempo-real.

(xi) Optimización del funcionamiento. Con base en la simulación anterior se optimizan los parámetros del robot y de sus componentes. Si las pruebas arrojan resultados que predicen el buen comportamiento de los componentes y satisfacen los requerimientos funcionales, significa que puede desarrollarse la tercera tarea de la metodología sin abandonar completamente las dos anteriores.

\section{Integración}

La integración significa el acoplamiento de todos los sistemas mecánicos, eléctricos, electrónicos y computarizados para configurar una nueva entidad mecatrónica con características definidas.

(xii) Integración del sistema mecatrónico. En esta tarea se integran el hardware del sistema mecánico + el hardware del sistema eléctrico / electrónico + el hardware del sistema de control y programación para obtener una nueva entidad física mecatrónica que cumpla con los requerimientos establecidos cuando se inicia el proyecto. El sistema de control depende del tamaño y sofisticación del sistema robotizado y puede seleccionarse un microcomputador PC, un PLC, un microcontrolador o un microprocesador, según objetivos del proyecto mecatrónico.

(xiii) Optimización y prueba del prototipo. En este paso se ajusta y optimiza el hardware del sistema mecatrónico. Su ciclo de vida depende del ciclo de vida de sus componentes. La prueba del prototipo abarca el cálculo de la resolución de control $\mathrm{CR}$, la precisión y la repetibilidad. Con esta prueba se determinan las características funcionales del prototipo.

\section{E. Identificar una necesidad}

En ingeniería, la necesidad de creación de productos y procesos eficientes es inevitable, la competencia entre las industrias obliga a buscar mejores procesos durante la producción. Los sistemas de manufactura integrados por computador (CIM 
Computer Integrated Manufacturing) mejoran la productividad de los procesos porque se realizan con mayor rapidez y exactitud evitando así los errores humanos. En los FMS cada una de las celdas realiza su función bajo control y programación de un computador digital dedicado; sin intervención del humano. El FMS de este proyecto está configurado con estación de carga/descarga robotizada, celda flexible de manufactura con dos máquinas CNC y robot serial de seis gof, celda de tratamientos térmicos con robot Cartesiano, celda de inspección y control de calidad con sistema de visión 3D y brazo-robot Mitsubishi de cinco gof, celda flexible de ensamblaje con brazo robot hidráulico de cuatro gof, y celda AS/ RS con sistema de visión 3D y brazo robot cartesiano objeto de este proyecto. Este trabajo dará la solución parcial a la celda AS/RS pues configura y diseña el robot Cartesiano que integra la celda flexible AS/RS al FMS.

\section{F. Morfología del robot}

La morfología del robot se refiere a la constitución física y composición identificada cada una de sus partes. La configuración de los robots industriales usualmente se asemeja al cuerpo humano, es decir, posee un cuerpo y un brazo, por lo general el cuerpo se encuentra en una parte fija o está montado sobre un riel y el brazo tiene la función de realizar las tareas ordenadas. Las partes que configuran un robot se pueden dividir en las siguientes: (1) Estructura mecánica (2) Transmisiones y reducciones (3) Sistemas de accionamiento (4) Sistema sensorial (5) Sistema de control (6) Elementos terminales.

\section{G. Descripción de la posición}

Los robots han de ser referenciados en el espacio tridimensional, es decir, que para poder localizar un cuerpo rígido en el espacio es necesario contar con una herramienta que permita la localización en el espacio por puntos. Un robot se encuentra referenciado en un sistema tridimensional y su posición está representada por un vector $\mathrm{P}^{\mathrm{M}}$ con tres posiciones con respecto a un sistema de referencia M, como se observa en la Figura 3. Para el análisis de la posición y orientación del robot, éste es representado como un cuerpo sólido; de tal manera que se pueda establecer un sistema de referencia mediante ejes ortogonales.

FIG. 3. Descripción de la posición

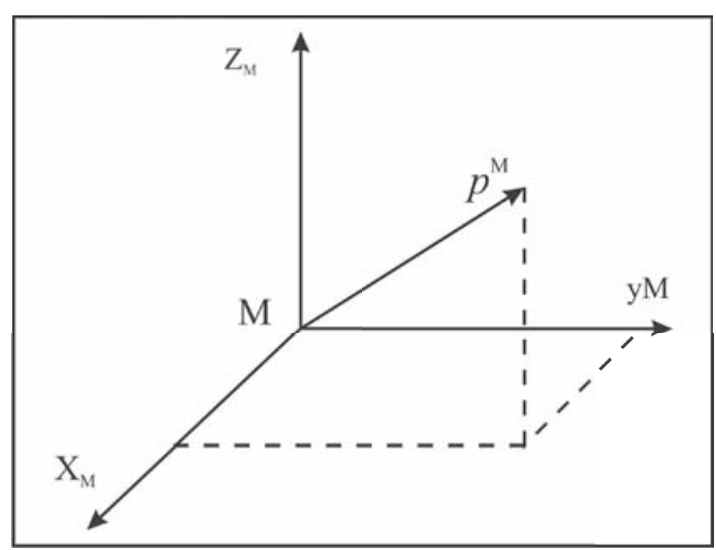

Fuente: Aparicio y orjuela [1], Godoy y Rodríguez [16]

Al tener asociado un objeto el sistema de referencia 0 , el vector $P_{O}^{M}$ representa la posición de origen del sistema $\mathrm{O}$ con respecto a $\mathrm{M}$. Como se observa en la Figura 4.

FIG. 4. Posición de un sistema o con respecto a otro m.

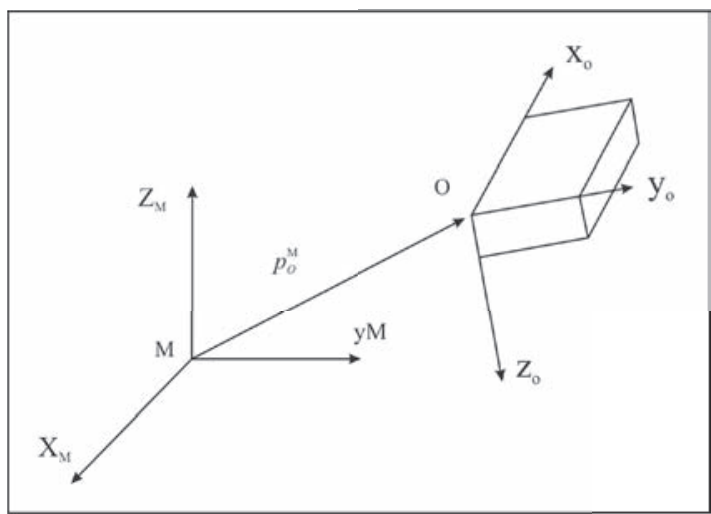

Fuente: Aparicio y orjuela [1], Godoy y Rodríguez [16]

Existen 3 métodos fundamentales para la representación de la posición, estos son:

Coordenadas cartesianas. El vector que la representa es el siguiente: $P_{O}^{M}(x, y, z)$

Coordenadas cilíndricas. El vector que la representa es el siguiente: $P_{O}^{M}(r, \Theta, z)$ 
Coordenadas esféricas. El vector que la representa es el siguiente: $P_{O}^{M}(r, \Theta, \phi)$

\section{H Descripción de la orientación}

Para un robot no es suficiente conocer e identificar su posición, es indispensable conocer y determinar su orientación específicamente del elemento terminal (EOA End Of Arm) el cual es el que interactúa con los demás objetos. Una orientación en el espacio tridimensional viene definida por tres grados de libertad o tres componentes linealmente independientes. Mientras que la posición de un cuerpo rígido respecto a un sistema de referencia $\mathrm{M}$ viene dada por la posición del origen del sistema de referencia $\mathrm{O}$ asociado a este, la orientación del cuerpo con respecto a un sistema de referencia M está dada por la orientación relativa de los ejes del sistema de referencia $\mathrm{O}$ asociado a este con respecto al sistema M. Esto se puede observar en la Figura 5.

FIG. 5. Sistemas de referencia coincidentes en el origen.

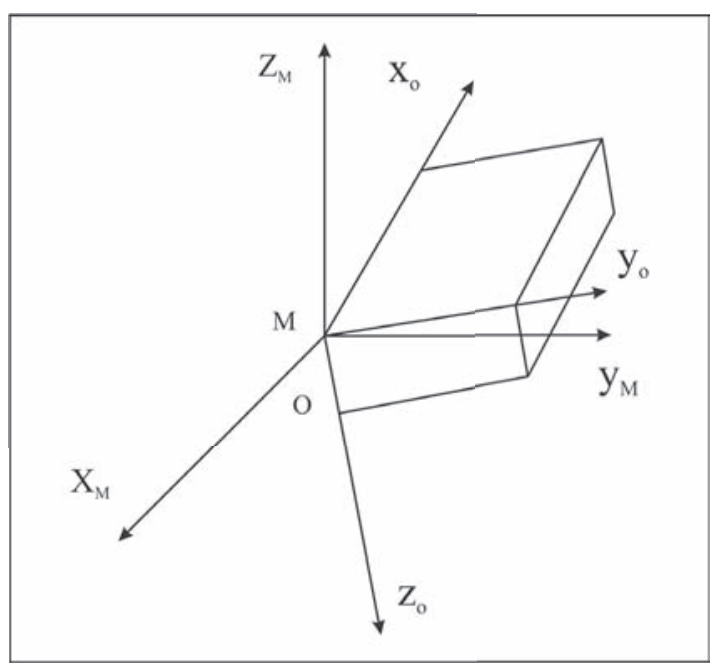

Fuente: Aparicio y Orjuela [1], Godoy y Rodríguez [16]

Las matrices de rotación son la metodología más utilizada para realizar la descripción de la orientación. Se define matriz de transformación aquella que opera sobre un vector de posición en un espacio tridimensional que se encuentra en un sistema de referencia rotado ouvw (sistema móvil) a un sistema de coordenadas de referencia fijo oxyz. Un vector que se encuentre en el plano se puede representar de la siguiente forma:

$$
\begin{aligned}
& P_{x y z}=\left[p_{x}, p_{y}, p_{z}\right]^{T}=p_{x} \cdot i_{x}+p_{y} \cdot j_{y}+p_{z} \cdot k_{z} \\
& P_{u w w}=\left[p_{u}, p_{v}, p_{w}\right]^{T}=p_{u} \cdot i_{u}+p_{v} \cdot j_{v}+p_{w} \cdot k_{w}
\end{aligned}
$$

Realizando una serie de transformación se obtiene:

$$
\begin{gathered}
{\left[\begin{array}{l}
p_{x} \\
p_{y} \\
p_{w}
\end{array}\right]=R\left[\begin{array}{l}
p_{u} \\
p_{v} \\
p_{w}
\end{array}\right]} \\
R=\left[\begin{array}{lll}
i_{x} i_{u} & i_{x} j_{v} & i_{x} k_{w} \\
j_{y} i_{u} & j_{y} j_{v} & j_{y} k_{w} \\
k_{z} i_{u} & k_{z} j_{v} & k_{z} k_{w}
\end{array}\right]
\end{gathered}
$$

R es la matriz de rotación que define la orientación del sistema ouvw con respecto al sistema oxyz, esta matriz es ortogonal y por lo tanto la inversa $\mathrm{R}^{-1}$ es igual a la traspuesta $\mathrm{R}^{\mathrm{T}}$ : $\mathrm{R}^{-1}=\mathrm{R}^{\mathrm{T}}$. Con esta matriz se realiza la representación de la orientación de sistemas girados únicamente sobre uno de sus ejes principales. Las siguientes matrices representan las rotaciones sobre los ejes xyz

$$
\begin{aligned}
R(x, \alpha) & =\left[\begin{array}{ccc}
1 & 0 & 0 \\
0 & \cos \alpha & -\sin \alpha \\
0 & \sin \alpha & \cos \alpha
\end{array}\right] \\
R(y, \alpha) & =\left[\begin{array}{ccc}
\cos \alpha & 0 & \sin \alpha \\
0 & 1 & 0 \\
-\sin \alpha & 0 & \cos \alpha
\end{array}\right] \\
R(z, \alpha) & =\left[\begin{array}{ccc}
\cos \alpha & -\sin \alpha & 0 \\
\sin \alpha 0 & \cos \alpha & 0 \\
0 & 0 & 1
\end{array}\right]
\end{aligned}
$$

\section{Coordenadas y matrices de transformación homogéneas MTH}

Para la posición y orientación conjuntamente de un objeto en el espacio se utilizan las MTH que son cuadradas $4 \mathrm{X} 4$ y compuesta por 4 submatrices de distinto tamaño. Una submatriz $\mathrm{R}_{3 \times 3}$ que representa la rotación, una submatriz $\mathrm{P}_{3 \times 1}$ que corresponde al vector de traslación, una submatriz $\mathrm{f}_{1 \times 3}$ que representa una transformación de perspectiva y una submatriz $\mathrm{W}_{1 \times 1}$ que representa un escalar.

$$
T=\left[\begin{array}{cc}
R_{3 \times 3} & P_{3 \times 1} \\
f_{1 \times 3} & W_{1 \times 1}
\end{array}\right]
$$

Para la configuración cartesiana en robótica sólo se realiza movimientos traslacionales, la representación en MTH se muestra en 8.0

$$
T=\left[\begin{array}{cccc}
1 & 0 & 0 & P_{x} \\
0 & 1 & 0 & P_{y} \\
0 & 0 & 1 & P_{z} \\
0 & 0 & 0 & 1
\end{array}\right]
$$


Ahora, si se desea conocer las componentes de un vector $r_{u v w}$ trasladado se obtiene de la siguiente forma:

$$
\left[\begin{array}{c}
r_{x} \\
r_{y} \\
r_{z} \\
1
\end{array}\right]=\left[\begin{array}{cccc}
1 & 0 & 0 & P_{x} \\
0 & 1 & 0 & P_{y} \\
0 & 0 & 1 & P_{z} \\
0 & 0 & 0 & 1
\end{array}\right]\left[\begin{array}{c}
r_{u} \\
r_{v} \\
r_{w} \\
1
\end{array}\right]
$$

\section{J. Modelo CAD d el r obot C artesiano P PP.P}

Los robots de configuración cartesiana son los adecuados para aplicaciones de almacenaje, según el tipo de almacenaje que se realizara en el módulo AS/RS; en el cual los pallet se almacenan en un rakc vertical, la configuración del robot cartesiano ideal para este tipo de aplicación es la vertical. Debe poseer 3 articulaciones prismáticas en la estructura y una rotacional en el elemento terminal. Los eslabones $X$ y $Z$ son perfiles rectangulares, que en su interior almacenan sus sistemas de transmisión. El modelo CAD de la estructura del robot se realizó mediante software especializado solid edge, se modelaron cada una de las partes del robot, cada uno de los eslabones completamente, los actuadores y la herramienta del elemento terminal. Una vez modelado cada uno de los eslabones del robot se integran las partes, convirtiendo el robot cartesiano como un conjunto final modelo CAD, como se muestra en la Figura 6 y 7.

FIG. 6. Modelo cad del robot ppp.

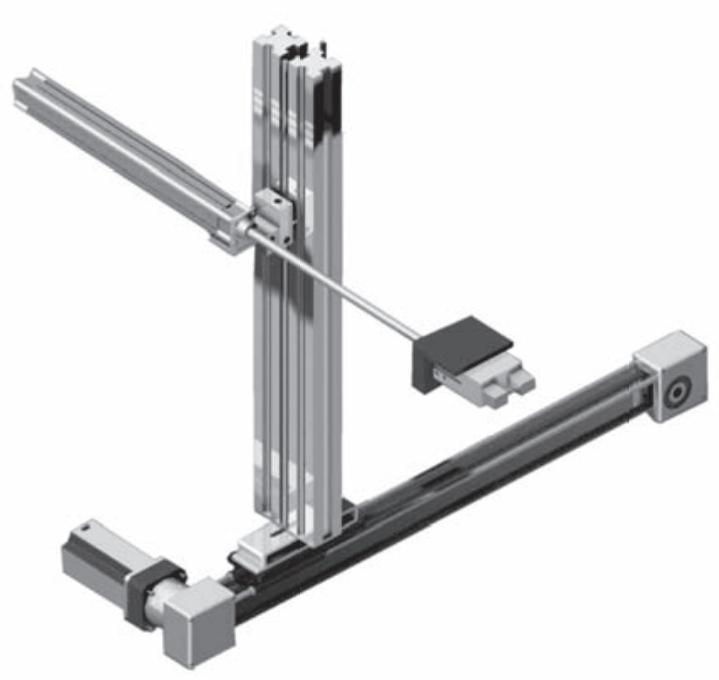

Fuente: R. Godoy y rodríguez [16]
FIG. 7. Modelo cad del robot PPP.R Y AS/RS

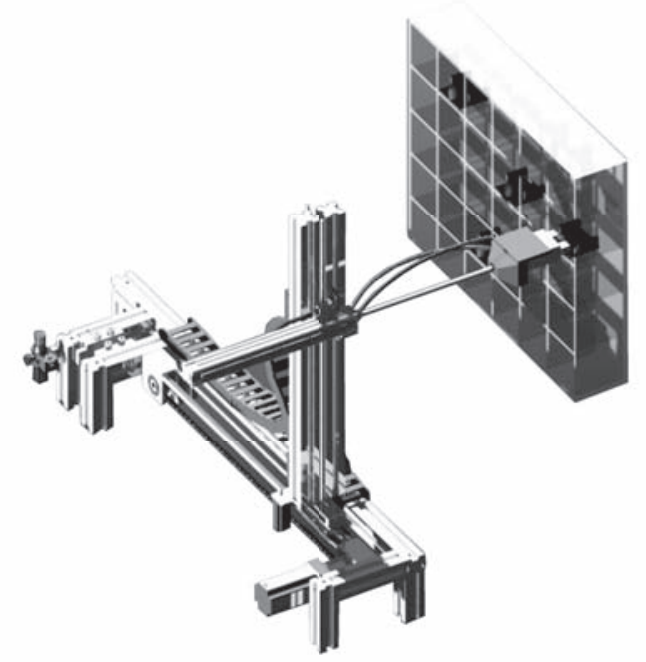

Fuente: Godoy y Rodríguez [16]

\section{K. Modelo de la cinemática directa}

La cinemática estudia el movimiento del robot con respecto al sistema de referencia y permite la identificación de la posición y orientación del elemento Terminal sin considerar las fuerzas o momentos que originan el movimiento. La cinemática directa determina la posición del elemento final con respecto al sistema de referencia, conocidos los valores de las articulaciones y los parámetros geométricos del robot. Como los elementos del robot se trasladan con respecto a un sistema de coordenadas de referencia, se debe establecer un sistema de referencia ligado a cada uno de los eslabones en movimiento, mediante el problema cinemática directa se busca establecer o determinar una MTH que permita relacionar el sistema de coordenadas ligado a cada eslabón con respecto al sistema de coordenadas de referencia. Existen varios métodos para encontrar esta matriz, uno de ellos es el método desarrollado por DenavitHartenberg. Con este método se relacionan los parámetros relativos al tamaño y la forma del eslabón $(a, \alpha)$ y los parámetros que relacionan la posición relativa de un eslabón con respecto a su eje $(d, \Theta)$.

\section{Metodología de Denavit-Hartenberg}

Para el desarrollo de esta metodología se define el origen de los sistemas de coordenadas de las 
articulaciones. Se denota el eje i como el eje de conexión de los elementos i-1 a i; para definir el marco del elemento i. La metodología para la cinemática directa basada en la sistemática Denavit- Hartenberg, se desarrolla en 9 pasos los cuales se describen a continuación:

1. Definir las direcciones de los ejes $Z 0, Z 1, \ldots$ Zn-1

2. Definir el origen Oo del sistema de coordenadas de la base del eje ZO

3. Definir el origen Oi en la intersección Zi con la normal común entre los ejes Zi-1 y Zi

4. Escoger el eje Xi a lo largo de la normal común a los ejes Zi-1 y Zi con dirección de la articulación i+1

5. Escoger el eje Yi, para que con Xi se cumpla la regla de la mano derecha.

6. Escoger el sistema de coordenadas de la herramienta donde $\mathrm{Xn}$ sea normal a Zn-1

7. Para $i=1,2, \ldots . n$ construir una tabla con los parámetros de ai, di, $\alpha i$, Qi

8. Con estos parámetros se calculan las matrices de transformación homogéneas.

9. Una vez obtenidos los parámetros representar el modelo de la cinemática directa para la posición y orientación del sistema de coordenadas de la herramienta.

En la Figura 8 se establecen los sistemas de coordenadas para el robot cartesiano PPP.

Una vez determinados los marcos de los elementos, la posición y orientación de cada uno de ellos, es posible establecer los parámetros:

$\alpha_{i}$ : Ángulo entre los ejes $Z_{i-1}$ y $Z_{i}$ alrededor del eje $X_{i}$, puede tomarse positivo cuando la rotación se hace en sentido horario

$\mathrm{Q}_{\mathrm{i}}$ : Ángulo entre los ejes $\mathrm{X}_{\mathrm{i}-1}$ y $\mathrm{X}_{\mathrm{i}}$ alrededor de $\mathrm{Z}_{\mathrm{i}-1}$, puede tomarse positivo cuando la rotación se hace en sentido horario

$\mathrm{d}_{\mathrm{i}}$ : Coordenada de $\mathrm{O}_{\mathrm{i}}$, a lo largo de $\mathrm{Z}_{\mathrm{i}-1}$

$a_{i}$ : Distancia entre $O_{i}$ y $O_{i}$ a lo largo de $X_{i-1}$

$Y$ se definen los parámetros Denavit-Hartenberg que representan la cinemática del robot:
FIG. 8. Metodología denavit- hartenberg [16

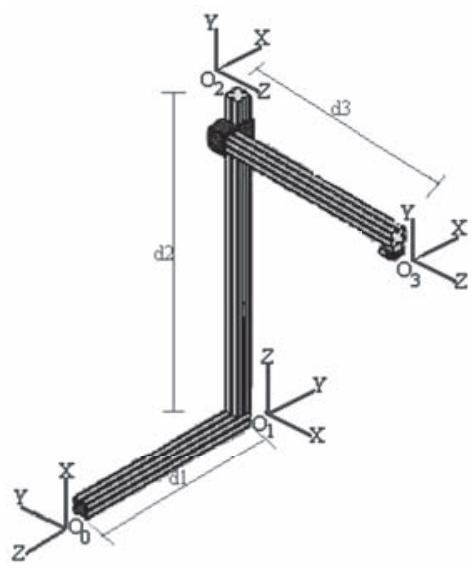

Fuente: Los autores

TABLA 1. PARÁMETROS DENAVIT-HARTENBERG [16]

\begin{tabular}{|c|c|c|c|c|}
\hline Eslabón & $\alpha_{\mathrm{i}}$ & $\Theta_{\mathrm{i}}$ & $\mathrm{d}_{\mathrm{i}}$ & $a_{\mathrm{i}}$ \\
\hline 1 & 90 & 90 & $\mathrm{~d} 1$ & 0 \\
\hline 2 & 90 & -90 & $\mathrm{~d} 2$ & 0 \\
\hline 3 & 0 & 0 & $\mathrm{~d} 3$ & 0 \\
\hline
\end{tabular}

Fuente: Los autores

\section{Cinemática directa}

Con la ecuación 10 se obtienen las matrices homogéneas para cada eslabón:

$$
\begin{aligned}
T & =A_{1}^{0} A_{2}^{1} A_{3}^{2} \\
A_{i}^{i-1} & =\left[\begin{array}{cccc}
\cos \Theta_{i} & -\cos \alpha_{i} \sin \Theta_{i} & \sin \alpha_{i} \sin \Theta_{i} & a_{i} \cos \Theta_{i} \\
\sin \Theta_{i} & \cos \alpha_{i} \cos \Theta_{i} & -\sin \alpha_{i} \cos \Theta_{i} & a_{i} \sin \Theta_{i} \\
0 & \sin \alpha_{i} & \cos \alpha_{i} & d_{i} \\
0 & 0 & 0 & 1
\end{array}\right]
\end{aligned}
$$

$A_{1}^{0}=\left[\begin{array}{cccc}0 & 0 & 1 & 0 \\ 1 & 0 & 0 & 0 \\ 0 & 1 & 0 & d_{1} \\ 0 & 0 & 0 & 1\end{array}\right]$

$A_{2}^{1}=\left[\begin{array}{cccc}0 & 0 & -1 & 0 \\ -1 & 0 & 0 & 0 \\ 0 & 1 & 0 & d_{2} \\ 0 & 0 & 0 & 1\end{array}\right]$

$A_{3}^{2}=\left[\begin{array}{cccc}1 & 0 & 0 & 0 \\ 0 & 1 & 0 & 0 \\ 0 & 0 & 1 & d_{3} \\ 0 & 0 & 0 & 1\end{array}\right]$ 


$$
T=\left[\begin{array}{cccc}
0 & 1 & 0 & d_{2} \\
0 & 0 & 1 & -d_{3} \\
1 & 0 & 0 & d_{1} \\
0 & 0 & 0 & 1
\end{array}\right]
$$

La matriz 14 define la posición y orientación del sistema de coordenadas de EOA con respecto al sistema de coordenadas de la base del robot. El correcto desarrollo de la anterior expresión se puede comprobar en la construcción del robot, en caso de errores se utiliza una matriz de ajuste.

\section{N. Cinemática inversa}

La cinemática inversa encuentra los valores de las articulaciones del robot para una posición y orientación específica de su EOA. La metodología para resolver este problema a partir de la MTH se describe a continuación:

Obtener la matriz de transformación homogénea T.

Para este caso particular se tiene que $T=A_{1}^{0} A_{2}^{1} A_{3}^{2}$ . Una vez obtenida esta matriz se procede a determinar la inversa de cada una de las matrices

$A_{i}^{i-1}$, para estos casos la inversa está dada por la siguiente matriz:

$$
\begin{aligned}
& \left(A_{i}^{i-1}\right)^{-1}=\left[\begin{array}{cccc}
n_{x} & s_{x} & a_{x} & -n^{T} P_{x} \\
n_{y} & s_{y} & a_{y} & -s^{T} P_{y} \\
n_{z} & s_{z} & a_{z} & -a^{t} P_{z} \\
0 & 0 & 0 & 1
\end{array}\right] \\
& \left(A_{1}^{0}\right)^{-1}=\left[\begin{array}{llll}
0 & 1 & 0 & 0 \\
0 & 0 & 1 & 0 \\
1 & 0 & 0 & -d_{1} \\
0 & 0 & 0 & 1
\end{array}\right] \\
& \left(A_{2}^{1}\right)^{-1}=\left[\begin{array}{llll}
0 & 1 & 0 & 0 \\
0 & 0 & 1 & 0 \\
1 & 0 & 0 & -d_{2} \\
0 & 0 & 0 & 1
\end{array}\right] \\
& \left(A_{3}^{2}\right)^{-1}=\left[\begin{array}{llll}
1 & 0 & 0 & 0 \\
0 & 1 & 0 & 0 \\
0 & 0 & 1 & -d_{3} \\
0 & 0 & 0 & 1
\end{array}\right]
\end{aligned}
$$

Para determinar la cinemática inversa se utiliza el siguiente procedimiento desde la ecuación 19:

$$
\begin{aligned}
& \left(A_{1}^{0}\right)^{-1} T=A_{2}^{1} A_{3}^{2} \\
& \left(A_{1}^{0}\right)^{-1}\left(A_{2}^{1}\right)^{-1} T=A_{3}^{2}
\end{aligned}
$$

La configuración del robot cartesiano no presenta alto grado de complejidad para determinar la cinemática inversa, simplemente es necesario saber cuáles son las coordenadas en el plano cartesiano en las cuales se encuentra ubicado el objeto y con estos valores se determina qué distancia se debe desplazar cada uno de los eslabones.

\section{O Modelo de la dinámica}

Ahora, se diseña y analiza la estructura mecánica del robot, se determina el tamaño de los motores y de los sistemas de transmisión. Existen tres metodologías que permiten desarrollar el modelo matemático de la dinámica de un robot industrial: La metodología de Newton-Euler, la metodología de Lagrange - Euler y el modelo matemático de Gibb. En este caso se usa la metodología de Lagrange-Euler porque las expresiones resultantes son más compactas con respecto a las obtenidas con la metodología Newton-Euler, porque en la formulación Lagrange-Euler no se consideran todas las acciones que no generen trabajo, además con esta metodología se obtiene el modelo dinámico en términos de trabajo y de energías.

La metodología de Lagrange-Euler analiza el sistema mecánico como un todo desde el punto de vista energético. En el proceso de su obtención se determina un conjunto de coordenadas generalizadas, es decir, se escogen sólo las variables de interés, o sea, la posición de cada unión, Barrientos y colaboradores, [2] Mendoza [10]. La formulación de Lagrange-Euler se expresa de la siguiente forma:

$$
\begin{aligned}
& \frac{d}{d t} \frac{\partial L}{\partial \dot{q}}-\frac{\partial L}{\partial q}=\tau \\
& L=K-P
\end{aligned}
$$

Donde:

$\mathrm{L}=$ Representa el Lagrangiano y es la diferencia entre la energía cinética y la energía potencial.

$\mathrm{K}=$ Energía cinética del robot.

$\mathrm{P}=$ Energía potencial del robot. 
$q=$ Variables de la articulación compuestas por ángulos $\Theta_{\mathrm{i}}$ ó desplazamientos $\mathrm{d}_{\mathrm{i}}$.

$\tau=$ Es el vector con componente $\mathrm{T}_{\mathrm{i}}$ de torques de los ángulos de la s articulaciones y $\mathrm{F}_{\mathrm{i}}$ de los desplazamientos en las articulaciones.

En la Figura 9 se representan las masas y las velocidades del robot Cartesiano. Se puede identificar cada una de sus masas y sus respectivas velocidades. Las velocidades y aceleraciones del robot se desarrollan a continuación.

FIG. 9. Representación de las masas y las velocidades

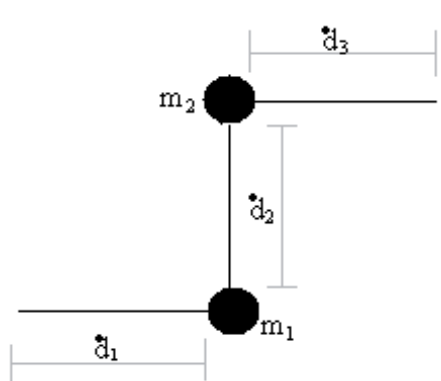

Fuente: Los autores

$$
\begin{aligned}
& K_{1}=\frac{1}{2}\left(m_{1}+m_{2}\right) \dot{d}_{1}^{2} \\
& P_{1}=0 \\
& K_{2}=\frac{1}{2} m_{2} \dot{d}_{3}^{2}
\end{aligned}
$$

Para la obtención del langrangiano se reemplazan los valores respectivos en la siguiente ecuación:

$$
\begin{gathered}
\mathrm{L}=\left(\mathrm{K}_{1}+\mathrm{K}_{2}\right)-\left(\mathrm{P}_{1}+\mathrm{P}_{2}\right) \\
L=\left(\frac{1}{2}\left(m_{1}+m_{2}\right) \dot{d}_{1}^{2}+\frac{1}{2} m_{2} \dot{d}_{3}^{2}\right)-\left(0+m_{2} g d_{2}\right)
\end{gathered}
$$

Aplicando la ecuación 15.0 sobre 22.0 se puede determinar la matriz que representa la dinámica del robot:

$$
\begin{aligned}
& \frac{\partial L}{\partial \dot{d}_{1}}=\left(m_{1}+m_{2}\right) \dot{d}_{1} \\
& \frac{d}{d t} \frac{\partial L}{\partial \dot{d}_{1}}=\left(m_{1}+m_{2}\right) \ddot{d_{1}} \\
& \frac{\partial L}{\partial d_{1}}=0 \\
& F_{1}=\left(m_{1}+m_{2}\right) \ddot{d}_{1}
\end{aligned}
$$

$$
\begin{aligned}
& \frac{\partial L}{\partial \cdot}=0 \\
& \partial d 2 \\
& \frac{d}{d t} \frac{\partial L}{\partial \dot{d}_{2}}=0 \\
& \frac{\partial L}{\partial d_{2}}=-m_{2} g \\
& F_{2}=m_{2} g \\
& \frac{\partial L}{\partial \dot{d}_{3}}=m_{2} \dot{d}_{3} \\
& \frac{d}{d t} \frac{\partial L}{\partial \dot{d}_{3}}=m_{2} \ddot{d}_{3} \\
& \partial L=0 \\
& F_{3}=m_{2} \ddot{d}_{3} \\
& {\left[\begin{array}{l}
F_{1} \\
F_{2} \\
F_{3}
\end{array}\right]=\left[\begin{array}{ccc}
\left(m_{1}+m_{2}\right) \ddot{d}_{1} & 0 & 0 \\
0 & m_{2} g & 0 \\
0 & 0 & m_{2} \ddot{d}_{3}
\end{array}\right]}
\end{aligned}
$$

\section{P Cálculo de fuerza en las articulaciones}

La expresión (32) representa el modelo dinámico del robot, se reemplazan los valores correspondientes para determinar la fuerza requerida para movilizar cada uno de los eslabones y definir el tamaño de los actuadores.

$$
\left[\begin{array}{l}
F_{1} \\
F_{2} \\
F_{3}
\end{array}\right]=\left[\begin{array}{ccc}
\left(m_{1}+m_{2}\right) \ddot{d}_{1} & 0 & 0 \\
0 & m_{2} g & 0 \\
0 & 0 & m_{2} \ddot{d}_{3}
\end{array}\right]
$$

Donde:

$m_{1}$ es la masa del eslabón 2, del conjunto del tornillo junto con la carcasa, es decir:

$$
\begin{aligned}
& m_{1} \approx 1,034 \mathrm{Kg}+0.4 \mathrm{Kg} \\
& m_{1}=1.434 \mathrm{Kg}
\end{aligned}
$$

$m_{2}$ es la masa del eslabón 3, junto con la carga que se va a transportar, es decir:

$$
\begin{aligned}
& m_{2}=2.6 \mathrm{Kg}+0.35 \mathrm{Kg}+0.42 \mathrm{Kg} \\
& m_{1}=3.37 \mathrm{Kg}
\end{aligned}
$$

$\ddot{d}_{1}$ y $\ddot{d}_{3}$ representan la aceleración de cada uno de los eslabones, en el diseño de se determina que la aceleración deseada en cada eslabón es 
igual y corresponde a $0.2 \mathrm{~m} / \mathrm{s}^{2}$. Por lo tanto se tiene que:

$$
\begin{aligned}
& {\left[\begin{array}{l}
F_{1} \\
F_{2} \\
F_{3}
\end{array}\right]=\left[\begin{array}{ccc}
(1.43+3.37) 9,8 & 0 & 0 \\
0 & 3.37 \times 9.8 & 0 \\
0 & 0 & 3.37 \times 0.2
\end{array}\right]} \\
& {\left[\begin{array}{l}
F_{1} \\
F_{2} \\
F_{3}
\end{array}\right]=\left[\begin{array}{c}
47.04 N \\
33.026 N \\
0.674 N
\end{array}\right]}
\end{aligned}
$$

\section{Q Diseño del sistema mecánico}

El robot tiene 3 grados de libertad, en el eje $X$ hay dos guías por las cuales se desplazará el eslabón vertical. La transmisión del movimiento en el eje $X$ se realiza mediante una correa dentada. Para el desplazamiento en el eje $Z$ se dispone de un tornillo de bolas y dos guías. El desplazamiento en el eje $Y$ se realiza utilizando como actuador un cilindro neumático. Para la estructura se selecciona aluminio porque posee buena resistencia mecánica, alta resistencia a la corrosión y poco peso. Adicionalmente, el aluminio es un material de bajo costo y abundante. El tipo de aluminio utilizado es una aleación 6061-T6.

El eslabón 1 se encuentra en el eje X y está compuesto por dos ejes paralelos sobre los cuales se desliza el vagón que sostiene el eslabón vertical, los dos ejes estas sujetos en cada uno de sus extremos por un soporte en aluminio, en el extremo izquierdo se ubicará el motor con su sistema de transmisión, a éste se sujetara la correa dentada la cual le transmitirá el movimiento al vagón. El eslabón 2 vertical se encuentra unido en la parte superior al vagón del eslabón 1 , y consta de un perfil rectangular hueco en el centro con el fin de reducir el peso de la estructura, permitir la ubicación de los sistemas de transmisión, los cables y los elementos electrónicos del robot. En el centro del perfil se encuentra un tornillo que transforma el movimiento rotacional a lineal del eslabón 3 que es un cilindro neumático, y se une al eslabón 2 en la tuerca del tornillo. En la Figura 13 se observa cada uno de los eslabones anteriormente nombrados. El robot posiciona los pallets en cada uno de los orificios del rack, por cada orificio se pueden introducir dos bandejas. De acuerdo con las dimensiones del rack se establecen las distancias máximas de desplazamiento para cada uno de los eslabones. Para el eslabón 1 la distancia que re- corre es de $80 \mathrm{~cm}$, para el eslabón 2 la distancia es de $60 \mathrm{~cm}$ y por último la distancia que recorre el eslabón 3 es de $40 \mathrm{~cm}$. El volumen ocupado por el eslabón 1 es de $382,80 \mathrm{~cm}^{3}$, la densidad del aluminio es de $0,00270 \mathrm{Kg} / \mathrm{cm}^{3}$, por lo tanto, la masa del perfil del elemento es: $\mathrm{mp}=1,034 \mathrm{Kg}$. El peso de la bandeja junto con la pieza con mayor peso que será transportada es de aproximadamente $420 \mathrm{~g}$ y el peso del cilindro es de $2600 \mathrm{~g}$, el peso de la herramienta es de aproximadamente 350g. $m_{B}=0.42 \mathrm{Kg}$ (masa de la bandeja junto con la pieza de mayor peso). $m_{c}=2.6 \mathrm{Kg}$ (masa del cilindro). $m_{H}=0.35 \mathrm{Kg}$ (masa de la herramienta)

\begin{tabular}{|c|c|c|}
\hline Elemento & Característica & Observación \\
\hline Eslabón 1 & $\begin{array}{c}\text { Sistema flexible } \\
\text { por correas } \\
\text { dentadas }\end{array}$ & $\begin{array}{l}\text { No contacto } \\
\text { metálico. No hay } \\
\text { deslizamiento. } \\
\text { No hay tensión. } \\
\text { No precisa } \\
\text { lubricación. } \\
\text { Mueve todo } \\
\text { el peso de la } \\
\text { estructura }\end{array}$ \\
\hline Eslabón 2 & $\begin{array}{c}\text { Sistema rígido } \\
\text { con tornillo de } \\
\text { bolas }\end{array}$ & $\begin{array}{l}\text { Longitud } 35.4 \\
\text { pulgadas. } \\
\text { Diámetro ext. } \\
0.375 \text { pulgadas. } \\
\text { Diámetro Int. } \\
0.360 \text { pulgadas. } \\
\text { Posición } \\
\text { vertical. } \\
\text { Coeficiente } \\
\text { de fricción } 1 . \\
\text { Carta } 13.628 \\
\text { Kg. Factor de } \\
\text { seguridad } 4\end{array}$ \\
\hline Eslabón 3 & $\begin{array}{c}\text { Cilindro } \\
\text { neumático }\end{array}$ & $\begin{array}{l}\text { Longitud de } \\
\text { carrera } 400 \\
\text { mm. Carga } \\
1.925 \mathrm{Kg} . \\
\text { Fuerza } 19 \mathrm{~N} \text {. } \\
\text { Presión de } \\
\text { trabajo } 6 \text { bar. } \\
\text { Diámetro de } \\
\text { embolo } 28 \mathrm{~mm}\end{array}$ \\
\hline
\end{tabular}

TABLA 2. ELEMENTOS DEL SISTEMA MECÁNICO 


\begin{tabular}{|c|c|c|}
\hline \multirow{4}{*}{ Motor 1 } & & Velocidad 95.5 \\
& Motor paso-a- & rpm. Torque 2.4 \\
& paso & 23.6 Watts. \\
& & Factor de \\
& & seguridad 2.5 \\
\hline \multirow{4}{*}{ Motor 2 } & & Velocidad \\
& & 3779.52 rpm. \\
& Motor paso-a- & Torque 0.7 Lb- \\
& pulg. Potencia \\
& & 41.9 Watts. \\
& & Factor de \\
& & seguridad 6.3 \\
\hline
\end{tabular}

Fuente: Los autores

\section{R Diseño de sistema electrónico}

Los motores paso a paso operan a 48V DC y se diseña un circuito de control de pulsos, como se muestra en la Figura 10, este circuito consta de un puente $\mathrm{H}$. El circuito tiene tres partes básicas. La parte A representa la entrada de los pulsos que moverán el motor, estos pulsos son generados por un circuito o por un microcontrolador, la velocidad del motor depende de la velocidad de generación de los pulsos, por tal motivo se diseña un generador de pulsos con un microcontrolador, esto con el fin de facilitar el control de la generación de los pulsos por medio de programación. La parte B es un circuito integrado puente $\mathrm{H}$, que es un arreglo de transistores que permite controlar motores por medio de señales de baja potencia provenientes de un circuito digital, y para este caso es el microcontrolador. La parte $\mathrm{C}$ es la salida del puente $\mathrm{H}$ estas llegan al motor para que realice el movimiento deseado.

FIG. 10. CIRCUITO BÁSICO PARA UN MOTOR PASO A PASO BIPOLAR

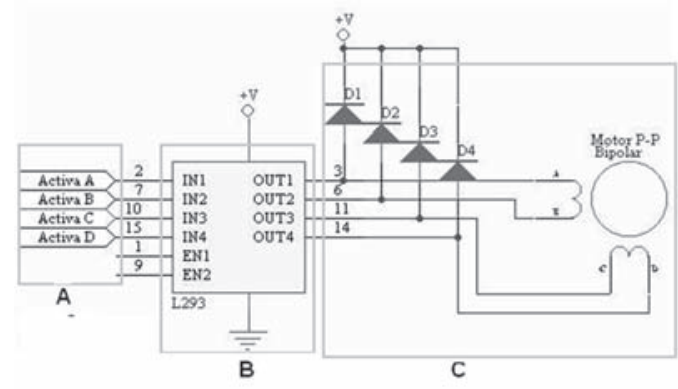

Fuente: Los autores
El diseño del circuito electrónico se muestra en la Figura 13, en donde el circuito digital es basado en microcontrolador y tiene como función entregar pulsos digitales al controlador de giro. El controlador de giro es un punto $\mathrm{H}$ encapsulado de referencia L293, en las salidas de este circuito integrado se adiciona un transistor de potencia, para aumentar la corriente que se entrega al motor y para evitar que se dañen los circuitos integrados. Dentro de este esquema también se dispone de la etapa de potencia y control de las electro válvulas. Este circuito no tiene gran predicción porque los componentes utilizados son dispositivos del mercado y por tanto son muy bajas, por tal motivo el controlador que se ajusta a las necesidades del diseño es el controlador para motores paso a paso de Festo tipo SEC-ST-48-6-P01. Este controlador tiene varias entradas las cuales permiten enviar señales desde un PLC porque integra los motores en un proceso de trabajo junto con un procesador central, que permite variar la velocidad del motor, controlar los voltajes de salida al motor al igual que su corriente y, además, por medio de entradas digitales, puede realizarse el control con respecto al número de pasos y el sentido mediante el microcontrolador mostrado en la Figura 11. Las características generales del controlador SEC-ST-48-6-P01.

FIG. 11. DISEÑO DEL CIRCUITO DE CONTROL

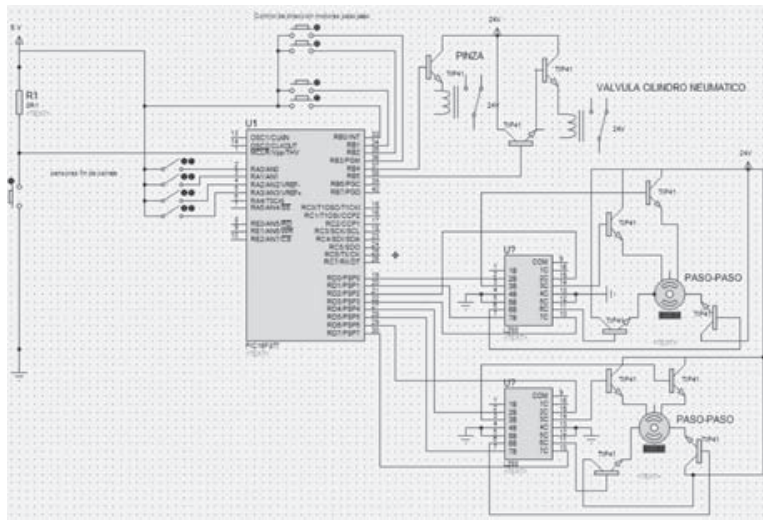

Fuente: Los autores

Los motores paso a paso tienen la ventaja de que no necesitan retroalimentación para obtener la información de la posición de su eje, esto elimina la necesidad de ubicar sensores que indique la posición final del motor, sin embargo, por seguridad se ubican sensores de final de carrera para evitar 
que los eslabones se pasen el límite de desplazamiento y los motores se dañen por exceso de fuerza.

\section{S Selección de muñeca y mecanismo de la garra}

Para el proyecto mecatrónico del mecanismo de la garra se integran los siguientes componentes: (1) estructura mecánica, (2) servomotor, (3) transmisión, (4) mecanismo de los dedos, (5) sensores y (6) controlador. La estructura es una pieza base para la instalación de los demás componentes. Los motores más empleados para accionar el mecanismo de los dedos son cilindros neumáticos y cilindros hidráulicos de hasta seis bares de presión, los motores paso-a-paso y servo motores eléctricos. Las transmisiones para el movimiento del mecanismo de los dedos son elementos mecánicos rígidos y flexibles. Los sensores son usados cuando se necesita controlar la presión de agarre. El controlador de la garra está centralizado e integrado al control central del robot para coordinar la apertura-cierre de los dedos y coordinar este movimiento con la cinemática del robot.

\section{T Mecanismos de manivela y guía}

Los mecanismos de manivela y guía tienen cuatro barras accionadas por un cilindro neumático o un cilindro hidráulico. Hay dos configuraciones comunes: cuando el mecanismo se acciona por la extensión del cilindro y cuando el mecanismo se acciona por la retracción del cilindro. La primera configuración se muestra en la Figura 12a. Cuando el cilindro ejecuta la carrera de avance entonces la mano se cierra. El movimiento hacia adelante del cilindro empuja los dos pequeños elementos hacia adelante. Los dos elementos más grandes rotan y forman los dedos de la garra con movimiento en arco alrededor del punto de pivote. Este mecanismo puede ejercer gran fuerza de sujeción. El incremento de la fuerza ocurre a medida que el ángulo A se aproxima a $90^{\circ}$, el cual es el punto en donde los dedos ajustan. Como este mecanismo es capaz de ejercer una gran fuerza, en el diseño es conveniente considerar el esfuerzo en los elementos. La otra variante del mecanismo, es cuando se acciona por la carrera de retracción del cilindro, en la Figura 12b, se ilustra esta situación en donde se emplea en el extremo de los dedos un bloque en $\mathrm{V}$ para levan- tar piezas cilíndricas. Los dedos son asistidos por resortes para prevenir que la pieza gire y salte de la garra. La fuerza de sujeción del mecanismo se calcula con la siguiente expresión:

$$
F_{0}=\frac{F_{i} \cdot D \cdot \operatorname{sen} B}{2 E \cdot \cos A}
$$

Donde:

$F_{0}$ : Fuerza de salida o de sujeción perpendicular al elemento $\mathrm{E}$.

$F_{i}$ : Fuerza de entrada ejercida por el cilindro.

A: Ángulo entre el elemento $\mathrm{C}$ y el vástago del cilindro.

$B$ : Ángulo entre el elemento $\mathrm{C}$ y el elemento $D$.

C,D,E : Longitudes de los elementos.

FIG. 12. MECANISMOS DE LOS DEDOS [3]

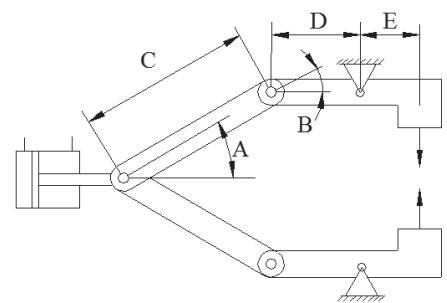

(a) Mecanismo de manivela y guia de extensión.

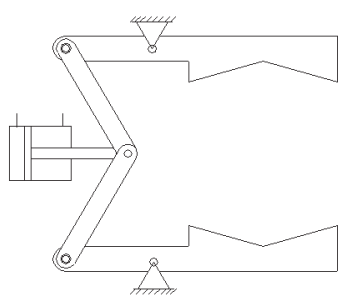

(b) Mecanismo de manivela y guia de retracción.

Fuente: Los autores

\section{CONCLUSIONES}

Se aplica una metodología de modelaje, diseño y simulación del movimiento en ambiente virtual orientada a la integración sinérgica de sistemas mecánicos, sistemas electrónicos y sistemas informáticos para configurar un nuevo sistema mecatrónico robotizado antes de desarrollarse en ambiente real. 


\section{REFERENCIAS}

[1] Aparicio González Marcela y Orjuela Parra Johann. Control Para Un Brazo Robot Colocado Sobre Una Plataforma Móvil “ÚRSULA”. Proyecto de Grado. Pontificia Universidad Javeriana. Facultad de Ingeniería. Carrera Ingeniería Electrónica. Bogotá, Colombia. 2005, 132p

[2] Barrientos Antonio, Luis Felipe Peñin, Carlos Balaguer y Rafael Aracil. Fundamentos de Robótica. Madrid, España. McGraw Hill, 2007, 624 p. ISBN978-84-4815636-7

[3] Carvajal Rojas J. H. Modelamiento y Diseño de Robots Industriales. Universidad de La Salle, Bogotá, Colombia. 2007, 390p. ISBN 958929074-4

[4] Evans, B. Simultaneous Engineering, Integrating Manufacturing and Design, SME. 1990.

[5] Groover M. P. Automation, Production Systems and Computer Integrated manufacturing. 2000. Prentice Hall, 856 p.ISBN 0-13-088978-4

[6] Groover, Mikell p. Robótica Industrial Tecnología, programación y aplicaciones. México. McGraw Hill, 1990, $600 \mathrm{p}$.

[7] Harley, J. \& Mortimer, J. Simultaneous Engineering. The Management Guide to Successful Implementation, Industrial Newsletters Ltda. 1991.

[8] Ishii, K. Modeling of Concurrent Engineering Design, Concurrente Engineering, Automation, Tools and Techniques, Wiley \& Sons. 1992.

[9] Hernán Felipe Rodríguez Garzón y Wilmar Giovanni Jiménez Sierra. Diseño y Simulación De Un Robot Cartesiano Para La Clasificación Y Almacenamiento De Piezas De La Estación CIM, Proyecto de Grado. Universidad De La Salle. Facultad de Ingeniería de Diseño y Automatización Electrónica. Bogotá, Colombia. 2006, $184 \mathrm{p}$.

[10] Mendoza José. Diseño Del Control De Un Robot De Dos Grados De Libertad Para Aplicaciones De Seguimiento De Objetos, Tesis. Instituto Nacional De Astrofísica, Óptica y Electrónica. Maestro en Ciencias en La especialidad de Electrónica. Tonantzintla, Mexico 2003, $156 p$

[11] Parsaei, H. R. \& Sullivan, W. G. Concurrente Engineering. Contemporary Issues and Modern Design Tools, Chapman and Hall. 1993.

[12] Shina, S. G. Concurrent Engineering and Design for Manufacturing of Electronic Products. Van Nostrand Reinhold. 1991.

[13] Shirley Joseph, Uicker John. Teoría de Máquinas y Mecanismos. México. McGraw Hill, 1988, 612p.
[14] World Robotics 2006

[15] Yazdani, B. Demystification of Concurrent Engineering. CARS \& FOF'96.

[16] Godoy Hernández R. D. y Rodríguez Quintero W. Diseño y Modelamiento de un Robot Cartesiano para el Posicionamiento de Piezas. Ingeniería de Diseño y Automatización Electrónica, Universidad de La Salle, 2008. Orientador: Jaime Humberto Carvajal Rojas. Evaluadores: Pedro Emilio Prieto y Jairo Orlando Montoya Gómez. 